\title{
ON INVARIANT SETS AND ON A THEOREM OF WAŻEWSKI ${ }^{1}$
}

\author{
PHILIP HARTMAN
}

\begin{abstract}
The first part of the paper treats the question of the existence of a solution $x=x(t)$ of an ordinary differential equation which exists for $t \geqq t_{0}$ and remains in a given closed set $F$ for every assigned initial point $\left(t_{0}, x\left(t_{0}\right)\right) \in F$ or, in the autonomous case, $x\left(t_{0}\right) \in F$. The results involve conditions which, for the autonomous case, reduce to $\operatorname{dist}\left(x^{0}+h f\left(x^{0}\right), F\right) / h \rightarrow 0$ as $h \rightarrow+0$ for all $x^{0} \in F$. The second part of the paper deals with theorems of the Ważewski type which, in some situations, permit the relaxation of the hypothesis that egress points are strict egress points.
\end{abstract}

1. Invariant sets. We shall first prove

THEOREM 1. Let $\Omega \subset E^{n}$ be open, $F \subset \Omega$ relatively closed in $\Omega$, and $f: \Omega \rightarrow E^{n}$ continuous. Then a necessary and sufficient condition that, for every $x_{0} \in F$, at least one solution $x=x(t)$ of the initial value problem

$$
x^{\prime}=f(x), \quad x(0)=x_{0},
$$

satisfies $x(t) \in F$ on its right maximal interval of existence is that

$$
d\left(x^{0}+h f\left(x^{0}\right), F\right) / h \rightarrow 0, \text { as } h \rightarrow+0, \text { for all } x^{0} \in F,
$$

where $d(x, F)=\inf |x-y|$ for $y \in F$.

The necessity of (2) is obvious. The converse becomes false if one replaces "at least one" by "every". This can be seen from the scalar example $x^{\prime}=x^{1 / 3}, \Omega=E^{1}, F=\{0\}$. A result corresponding to Theorem 1 was proved by Brezis [1] under the additional assumption that $f$ is locally uniformly Lipschitz continuous. His proof depended in an essential way on this assumption and is quite different from the proof of Theorem 1 below.

Proof. Assume (2). It is sufficient to prove the existence of a solution $x=x(t)$ of $(1), x(t) \in F$, on a small interval $[0, \alpha]$. Let $b>0$ be so small that the closed ball $\Sigma_{2 b}=\left\{x:\left|x-x_{0}\right| \leqq 2 b\right\}$ is in $\Omega$, and let $M$ satisfy $|f(x)| \leqq M$

Received by the editors June 7, 1971 and, in revised form, July 20, 1971.

AMS 1970 subject classifications. Primary 34C99, 34D99.

Key words and phrases. Invariant sets, trajectory derivative, Lyapunov, egress points.

${ }^{1}$ The study was supported by the Air Force Office of Scientific Research Contract No. F44620-67-C-0098. 
on $\Sigma_{2 b}$, and $\alpha=b / 2 M$. Any initial value problem

$$
x^{\prime}=f(x), \quad x\left(t^{0}\right)=x^{0} \in \Sigma_{b},
$$

has a solution $x=x^{0}(t), x^{0}(t) \in \Sigma_{2 b}$, on $\left[t^{0}, t^{0}+2 \alpha\right]$. Let $x^{0} \in F \cap \Sigma_{b}$ and for $t \in\left[t^{0}, t^{0}+\alpha\right]$, choose a $y_{t} \in F$ satisfying $d\left(x^{0}+\left(t-t^{0}\right) f\left(x^{0}\right), F\right)=\mid x^{0}+$ $\left(t-t^{0}\right) f\left(x^{0}\right)-y_{t} \mid$. Thus, as $t \rightarrow t^{0}+0$,

$$
\begin{aligned}
\left|y_{t}-x^{0}(t)\right| \leqq & \left|x^{0}(t)-x^{0}-\left(t-t^{0}\right) f\left(x^{0}\right)\right| \\
& +d\left(x^{0}+\left(t-t^{0}\right) f\left(x^{0}\right), F\right)=o\left(t-t^{0}\right) .
\end{aligned}
$$

Hence, as $t \rightarrow t^{0}+0$,

(4) $\left(y_{t}-x^{0}\right) /\left(t-t^{0}\right)=\left(x^{0}(t)-x^{0}\right) /\left(t-t^{0}\right)+o(1)=f\left(x^{0}\right)+o(1)$.

If $\varepsilon>0$ and $S>0$ is sufficiently small, then (3), (4) imply that the linear function $L(t)$ defined by

$$
L(t)=L\left(t ; x^{0}, t^{0}, S\right)=x^{0}+\left(t-t^{0}\right)\left(y_{t^{0}+S}-x^{0}\right) / S
$$

satisfies $L\left(t^{0}\right)=x^{0} \in F, L\left(t^{0}+S\right)=y_{t^{0}+S} \in F$ and

$$
\left|L^{\prime}(t)-f(L(t))\right|=\left|\left(y_{t^{0}+S}-x^{0}\right) / S-f(L(t))\right| \leqq \varepsilon \quad \text { on }\left[t^{0}, t^{0}+S\right] .
$$

Let $0<\varepsilon \leqq M$. Let $Q_{\varepsilon}$ denote the collection of pairs $(X, T)$, where $0<T \leqq \alpha$ and $X=X(t)$ is a function on the interval $[0, T]$ satisfying (i) $X(0)=x_{0} ; X(T) \in F$; (ii) $|X(t)-X(s)| \leqq 2 M|t-s|$ for $0 \leqq s, t \leqq T$; hence (iii) $\left|X(t)-x_{0}\right| \leqq 2 M T \leqq 2 M \alpha=b$ on $[0, T]$; (iv) $\left|X^{\prime}(t)-f(X(t))\right| \leqq 2 \varepsilon$ almost everywhere; finally, (v) every subinterval of $[0, T]$ of length $\geqq \varepsilon$ contains a point $t=t_{0}$ such that $X\left(t_{0}\right) \in F$.

The set $Q_{\varepsilon}$ is not empty. It contains, for example, the pair $(L, S)$, where $L$ is the linear function $L(t)=L\left(t ; x_{0}, 0, S\right)$ in (5) and $S>0$ is small. Introduce a partial ordering in $Q_{\varepsilon}$ as follows: $\left(X_{1}, T_{1}\right)<\left(X_{2}, T_{2}\right)$ if $T_{1}<T_{2}$ and $X_{2} \mid\left[0, T_{1}\right] \equiv X_{1}$. It is clear that any chain in $Q_{\varepsilon}$ possesses an upper bound in $Q_{\varepsilon}$. Thus, by Zorn's lemma, $Q_{\varepsilon}$ contains a maximal element $\left(X_{\varepsilon}, T_{\varepsilon}\right)$. Then $T_{\varepsilon}=\alpha$, for otherwise we can extend $X_{\varepsilon}(t)$ to an interval $\left[0, T_{\varepsilon}+S\right]$ as the linear function $L(t)=L\left(t ; X_{\varepsilon}\left(T_{\varepsilon}\right), T_{\varepsilon}, S\right)$ on $\left[T_{\varepsilon}, T_{\varepsilon}+S\right]$, where $S>0$ is sufficiently small.

By the Ascoli-Arzela theorem, there is a sequence $\varepsilon(1)>\varepsilon(2)>\cdots$ such that $\varepsilon(m) \rightarrow 0$ and $x(t)=\lim X_{\varepsilon(m)}(t)$ exists uniformly on $[0, \alpha]$, as $m \rightarrow \infty$. Clearly, $x(t)$ is a solution of $(1)$ and $x(t) \in F$ on $[0, \alpha]$.

This proof is also valid in the nonautonomous case and gives

THEOREM 2. Let $\Omega \subset E^{n+1}$ be open, $F \subset \Omega$ relatively closed in $\Omega$, and $f: \Omega \rightarrow E^{n}$ continuous. Then a necessary and sufficient condition that, for every $\left(t_{0}, x_{0}\right) \in F$, at least one solution $x=x(t)$ of the initial value problem

$$
x^{\prime}=f(t, x), \quad x\left(t_{0}\right)=x_{0},
$$


satisfies $(t, x(t)) \in F$ on its right maximal interval of existence is that

$$
d\left(\left(t^{0}+h, x^{0}+h f\left(t^{0}, x^{0}\right)\right), F\right) / h \rightarrow 0 \text {, as } h \rightarrow+0,
$$

for all $\left(t^{0}, x^{0}\right) \in F$.

Using this result, the smoothness conditions of Theorem 2 of Brezis [1] can be reduced accordingly. This involves a simplification, but no essential modification, of Brezis's proof. We indicate this by proving a related theorem.

CoRollary 1. Let $\Omega \subset E^{n+1}$ be open, $f: \Omega \rightarrow E^{n}$ continuous, $L^{k}: \Omega \rightarrow E^{1}$ of class $C^{1}$ for $1 \leqq k \leqq m$, and

$$
\dot{L}^{k}\left(t^{0}, x^{0}\right) \equiv L_{t}+L_{x} \cdot f \leqq 0 \quad \text { if }\left(t^{0}, x^{0}\right) \in \Pi^{k},
$$

where $L_{t}=\partial L / \partial t, L_{x}=\operatorname{grad}_{x} L$, and $L_{(t, x)}=\operatorname{grad}_{(t, x)} L$. Let $I=I\left(t^{0}, x^{0}\right)$ be the set of indices $k, 1 \leqq k \leqq m$, for which $\left(t^{0}, x^{0}\right) \in \Pi^{k}, \dot{L}^{k}\left(t^{0}, x^{0}\right)=0$ and, if I is not empty, suppose that either

$$
L_{(t, x)}^{k}\left(t^{0}, x^{0}\right), k \in I \text {, are linearly independent, }
$$

or that

$$
L^{k}\left(t^{0}+h, x^{0}+h f\left(t^{0}, x^{0}\right)\right) \leqq 0 \quad \text { for small } h \geqq 0 \text { and } k \in I .
$$

Then at least one solution $x=x(t)$ of the initial value problem

(12) $x^{\prime}=f(t, x), x\left(t_{0}\right)=x_{0}, \quad$ where $L^{k}\left(t_{0}, x_{0}\right) \leqq 0$ for $1 \leqq k \leqq m$, satisfies $L^{k}(t, x(t)) \leqq 0$ for $1 \leqq k \leqq m$ on its right maximal interval of existence.

A condition involving the alternative (10) or (11) cannot be omitted (even in the autonomous case), as can be seen from the example $n=2, x=$ $\left(x^{1}, x^{2}\right), f(t, x)=(1,0), m=2$, and $L^{1}(t, x)=x^{2}, L^{2}(t, x)=|x-(0,1)|^{2}-1$, so that $L^{1} \leqq 0, L^{2} \leqq 0$ only for $x=(0,0)$. But the solution $x=x(t)$ with the initial condition $x(0)=0$ is $x(t)=(t, 0)$. This example also shows that the condition involving (10) or (11) cannot be relaxed as follows: the index set $I \subset\{1, \cdots, m\}$ can be written as a disjoint union $I=I_{1} \cup I_{2}$ such that $L_{(t, x)}^{k}\left(t^{0}, x^{0}\right), k \in I_{1}$, are linearly independent and the inequality in (11) holds for $k \in I_{2}$. See Theorem 3 below if the set $F=\bigcap\left\{L^{k} \leqq 0\right\}$ is the closure of its interior.

Proof. Let $F=\left\{(t, x): L^{k}(t, x) \leqq 0\right.$ for $\left.1 \leqq k \leqq m\right\}$. In view of Theorem 2 , it suffices to verify (7) at a point, say $\left(t^{0}, x^{0}\right)=(0,0)$, such that $I=I(0,0)$ is not empty and $(10)$ holds. Let $I(0,0)=\{1, \cdots, p\}$, where $1 \leqq p \leqq m$, so that either $L^{k}(0,0)<0$ or $\dot{L}^{k}(0,0)<0$ for $k>p$. Let $\Delta_{k}=L_{(t, x)}^{k}(0,0)$ for $1 \leqq k \leqq p$ and $v=(1, f(0,0))$, so that $\Delta_{1}, \cdots, \Delta_{p}$ and $v$ are constant $(n+1)$-vectors satisfying $\Delta_{k} \cdot v=0$ and $\Delta_{1}, \cdots, \Delta_{p}$ are linearly independent. 
Let $\xi=(t, x)=\left(\xi^{1}, \cdots, \xi^{n+1}\right)$ and $\eta=\phi(\xi)$ a local $C^{1}$-diffeomorphism of the $\xi$-space into an $\eta$-space such that $\phi(0)=0$ and the Jacobian matrix $\phi_{*}^{0}=(\partial \phi(0) / \partial \xi)$ is the identity, so that $\Delta_{k}=\phi_{*}^{0} \Delta_{k}, v=\phi_{*}^{0} v$. It suffices to verify (7) at $\eta=0$ when $d((h, h f(0,0)), F)=d(v h, F)$ is replaced by $d(\phi(v h), \phi(F))$, i.e., if the line segment $\xi=h v$ for small $h \geqq 0$ is replaced by its image arc $C: \eta=\phi(h v)$ and $F$ by $\phi(F)$. Hence, it can be supposed that, in a neighborhood of $\eta=0$,

$$
\left\{\eta: L^{k} \leqq 0\right\} \equiv\left\{\eta: \Delta_{k} \cdot \eta \leqq 0\right\} \text { for } 1 \leqq k \leqq p,
$$

while $L^{k}(0,0)<0$ or $\dot{L}^{k}(0,0)<0$ for $k>p$. The condition $\Delta_{k} \cdot v=0$ means that $L^{k} \leqq 0$ on the line segment $\pi=\{\eta: \eta=h v$ for small $h \geqq 0\}, k \leqq p$. Clearly, if $k>p, L^{k} \leqq 0$ also holds on $\pi$ and hence $\pi \in \phi(F)$. Since $\pi$ is tangent to $C: \eta=\phi(h v)$ at $h=0,(7)$ holds at $\eta=0$.

COROllary 2. Let $\Omega \subset E^{n+1}$ be open, $F \subset \Omega$ a convex set relatively closed in $\Omega$, and $f: \Omega \rightarrow E^{n}$ continuous. Then a necessary and sufficient condition that, for every $\left(t_{0}, x_{0}\right) \in F$, at least one solution $x=x(t)$ of the initial value problem (6) satisfies $(t, x(t)) \in F$ on its right maximal interval of existence is that $\Delta \cdot\left(1, f\left(t^{0}, x^{0}\right)\right) \leqq 0$ for every $\left(t^{0}, x^{0}\right) \in \partial F \cap \Omega$ and every outward normal $\Delta$ to $F$ at $\left(t^{0}, x^{0}\right)$.

By an outward normal to $F$ at $\left(t^{0}, x^{0}\right) \in \partial F$ is meant an $(n+1)$-vector $\Delta$ satisfying $\Delta \cdot\left(t-t^{0}, x-x^{0}\right) \leqq 0$ for all $(t, x) \in F$. Corollary 2 follows from the fact that if $v$ is an $(n+1)$-vector and $\left(t^{0}, x^{0}\right) \in \partial F$, then $d\left(\left(t^{0}, x^{0}\right)+h v, F\right) / h$ $\rightarrow 0$ as $h \rightarrow+0$ if and only if $\Delta \cdot v \leqq 0$ for all outward normals $\Delta$ to $F$ at $\left(t^{0}, x^{0}\right)$. This can be verified by considering, for small $h>0$, the unique point $\left(t_{h}, x_{h}\right) \in F$ nearest to the point $\left(t^{0}, x^{0}\right)+h v$ and, if these points do not coincide, the unit vector $\Delta_{h}$ in the direction $\left(t^{0}, x^{0}\right)+h v-\left(t_{h}, x_{h}\right)$, so that $\Delta_{h}$ is an outward normal to $F$ at $\left(t_{h}, x_{h}\right)$.

REMARK. In Corollary 2, the condition that $F$ is convex can be relaxed to the condition that, for every $\left(t_{0}, x_{0}\right) \in F$, the intersection of $F$ and a small ball in $\Omega$ with $\left(t_{0}, x_{0}\right)$ as center is convex; correspondingly, the definition of outward normal can be "localized".

2. On a theorem of Ważewski. In this section, we obtain Theorems 3 and 4, analogues of a result of Ważewski. In these theorems, the set of egress (=strict egress) points is replaced by other sets (and, in particular, it is not required that an egress point be a strict egress point). Here, $k$, $\alpha, \beta$ denote indices on the ranges

$$
1 \leqq k \leqq p+q ; \quad 1 \leqq \alpha \leqq p ; \quad p+1 \leqq \beta \leqq p+q,
$$

where $p \geqq 0, q \geqq 0, p+q>0$ (with the understanding that $p=0$ or $q=0$ means that the indices $\alpha$ or $\beta$ do not occur). 
(H1) $\Omega \subset E^{n+1}$ is open and $f: \Omega \rightarrow E^{n}$ is continuous.

(H2) $L^{k}: \Omega \rightarrow E^{1}$ is of class $C^{1}$ with the properties that

$$
\Omega^{0}=\left\{(t, x) \in \Omega: L^{k}(t, x)<0 \text { for } 1 \leqq k \leqq p+q\right\}
$$

is a nonempty open set with a boundary $\bigcup \Pi^{k}$ relative to $\Omega$, where

$$
\Pi^{k}=\left\{(t, x) \in \Omega \cap \bar{\Omega}^{0}: L^{k}(t, x)=0 \text { and } L^{j}(t, x) \leqq 0\right.
$$

and that

$$
\text { for } 1 \leqq j \leqq p+q\} \text {, }
$$

$$
\dot{L}^{\alpha}(t, x) \geqq 0 \quad \text { on } \Pi^{\alpha}, \quad \dot{L}^{\beta}(t, x) \leqq 0 \quad \text { on } \Pi^{\beta} .
$$

Let $Q$ be the set

$$
Q=\bigcup_{\alpha} \Pi^{\alpha}-\bigcup_{\beta} \Pi^{\beta}
$$

and $S$ a nonempty compact set satisfying $S \subset \Omega^{0} \cup Q$, and $S \cap Q$ is not a retract of $S$ but is a retract of $Q$. (When strict inequalities hold in (3), $Q$ is the set of egress [=strict egress] points of $\Omega^{0}$ (Ważewski, cf. [2, Lemma 3.1, p. 281]).)

(H3) $I=I(t, x)$ is the set of indices $k$ (if any) such that $(t, x) \in \Pi^{k}$ and $\dot{L}^{k}(t, x)=0$. If $I(t, x)$ is not empty, then

$$
L_{(t, x)}^{k}(t, x), k \in I \text {, are linearly independent. }
$$

$\left(\mathrm{H}^{\prime}\right)$ Or, more generally,

$$
L_{(t, x)}^{k}(t, x) \neq 0 \text { for } k \in I(t, x) ;
$$

if $I_{a}=I_{a}(t, x), I_{b}=I_{b}(t, x)$ are the sets of indices $k=\alpha, \beta$ in $I(t, x)$ and if neither $I_{a}$ nor $I_{b}$ is empty, then

$$
\operatorname{span}\left\{L_{(t, x)}^{\alpha}(t, x), \alpha \in I_{a}\right\} \cap \operatorname{span}\left\{L_{(t, x)}^{\beta}(t, x), \beta \in I_{b}\right\}=\{0\} .
$$

TheOREM 3. Assume $\left(\mathrm{H}_{1}\right),\left(\mathrm{H}_{2}\right),\left(\mathrm{H}_{3}\right)$ or $\left(\mathrm{H}^{\prime}\right)$. Then there is a point $\left(t_{0}, x_{0}\right) \in S$ and a solution of the initial value problem

$$
x^{\prime}=f(t, x), \quad x\left(t_{0}\right)=x_{0},
$$

such that $(t, x(t)) \in \Omega^{0}$ on its right maximal interval of existence.

This is an analogue of a theorem of Ważewski (cf. e.g., [2, Corollary 3.1, p. 282]) in which strict inequalities are required in (3), so that $I(t, x)$ is empty for all $(t, x)$, and $\Omega^{0}$ is replaced by $\Omega^{0}$ in the conclusion. Actually, we shall deduce Theorem 3 from this result of Ważewski.

Lemma 1. Assume $(\mathrm{H} 1),(\mathrm{H} 2)$ and $\left(\mathrm{H}^{\prime}\right)$. Then there exist continuous $h_{0}: \Omega \rightarrow E^{1}, h: \Omega \rightarrow E^{n}$ with the properties that

$$
\left|h_{0}\right| \leqq \frac{1}{2}, \quad|h| \leqq \frac{1}{2}
$$


and, for $0<\varepsilon \leqq 1$,

$$
L^{\alpha^{*}}(t, x)>0 \quad \text { on } \Pi^{\alpha}, \quad L^{\beta^{*}}(t, x)<0 \quad \text { on } \Pi^{\beta},
$$

where the meaning of the asterisk is given by

$$
L^{*}=L_{t}\left(1+\varepsilon h_{0}\right)+L_{x} \cdot(f+\varepsilon h)=\dot{L}+\varepsilon\left(L_{t} h_{0}+L_{x} \cdot h\right),
$$

so that $L^{*}$ is the trajectory derivative of $L$ with respect to the autonomous system

$$
d x / d s=f(t, x)+\varepsilon h(t, x), \quad d t / d s=1+\varepsilon h_{0}(t, x) .
$$

Proof OF The Lemma 1. Let $c=a$ or $c=b$. If $I_{c}=I_{c}\left(t^{0}, x^{0}\right)$ is not empty, consider the cone

$$
C_{c}=\left\{v=\sum \lambda_{k} L_{(t, x)}^{k}\left(t^{0}, x^{0}\right): \lambda_{k} \geqq 0, k \in I_{c}\right\} .
$$

It follows from (6) that if $\left(t_{1}, x_{1}\right) \in \Omega^{0}$ is sufficiently near to $\left(t^{0}, x^{0}\right)$ and $w_{0}=\left(t_{1}-t^{0}, x_{1}-x_{0}\right)$, then

$$
w_{0} \cdot L_{(t, x)}^{k}\left(t^{0}, x^{0}\right)<0 \quad \text { for } k \in I\left(t^{0}, x^{0}\right) .
$$

Consider the section $S_{c}=\left\{v: v \cdot w_{0}=-1, v \in C_{c}\right\}$ of the cone $C_{c}$, so that $S_{c}$ is the convex hull of the set of vectors

$$
\left\{\Delta_{k}=-L_{(t, x)}^{k}\left(t^{0}, x^{0}\right) / w_{0} \cdot L_{(t, x)}^{k}\left(t^{0}, x^{0}\right), k \in I_{c}\right\} .
$$

Let $I_{c 0}$ be the set of indices $k \in I_{c}$ such that $\left\{\Delta_{k}: k \in I_{c 0}\right\}$ are the extreme points of $S_{c}$, so that if $v \in S_{c}$, then $v$ has a unique representation $v=\sum \lambda_{k} \Delta_{k}$, $k \in I_{c 0}, \lambda_{k} \geqq 0, \sum \lambda_{k}=1$. In particular, if $j \in I_{c 1} \equiv I_{c}-I_{c 0}$, then $L_{(t, x)}^{j}\left(t^{0}, x^{0}\right)$ has a representation of the form

$$
L_{(t, x)}^{j}\left(t^{0}, x^{0}\right)=\sum \lambda_{k} L_{(t, x)}^{k}\left(t^{0}, x^{0}\right), \quad k \in I_{c 0}, \lambda_{k} \geqq 0, \sum \lambda_{k}>0 .
$$

The vectors

$$
\Delta_{k}, k \in I_{c 0} \text {, are linearly independent. }
$$

For suppose that some nontrivial linear combination $\sum c_{k} \Delta_{k}=0$, and write

$$
\sum^{\prime} c_{k} \Delta_{k}=-\sum^{\prime \prime} c_{k} \Delta_{k},
$$

where $\Sigma^{\prime}, \Sigma^{\prime \prime}$ denote the sums over the indices $k \in I_{c 0}$ for which $c_{k} \geqq 0, c_{k}<0$. Multiplying (14) scalarly by $w_{0}$ gives $\Sigma^{\prime} c_{k}=-\Sigma^{\prime \prime} c_{k}>0$. If (14) is divided by this positive number, the result can be written in the form

$$
\sum^{\prime} \lambda_{k}^{\prime} \Delta_{k}=\sum^{\prime \prime} \lambda_{k}^{\prime \prime} \Delta_{k}, \quad \text { where } \lambda_{k}^{\prime}, \lambda_{k}^{\prime \prime} \geqq 0, \quad \sum^{\prime} \lambda_{k}^{\prime}=\sum^{\prime \prime} \lambda_{k}^{\prime \prime}=1 .
$$


But this contradicts that fact that $\left\{\Delta_{k}, k \in I_{c 0}\right\}$ is the set of extremal points of $S_{c}$.

When $I_{c}=I_{c}\left(t_{0}, x_{0}\right)$ is empty, let $I_{c 0}$ be the empty set. Put $I_{c 1}=I_{c}-I_{c 0}$, $I_{0}=I_{a 0} \cup I_{b 0}, I_{1}=I_{a 1} \cup I_{b 1}$ in any case, so that $I=I_{a} \cup I_{b}=I_{a 0} \cup I_{a 1} \cup I_{b 0} \cup I_{b 1}=$ $I_{0} \cup I_{1}$ are disjoint unions (of possibly empty sets). When $I\left(t^{0}, x^{0}\right)$ is not empty, it follows from (7) and (13) that

$$
L_{(t, x)}^{k}\left(t^{0}, x^{0}\right), k \in I_{0}=I_{0}\left(t^{0}, x^{0}\right), \text { are linearly independent. }
$$

Hence there exists a scalar $g_{0}\left(t^{0}, x^{0}\right)$ and an $n$-vector $g\left(t^{0}, x^{0}\right)$ satisfying

$$
L_{t}^{\alpha} g_{0}+L_{x}^{\alpha} \cdot g=1, \quad L_{t}^{\beta} g_{0}+L_{x}^{\beta} \cdot g=-1
$$

at $\left(t^{0}, x^{0}\right)$ for $\alpha, \beta \in I_{0}\left(t^{0}, x^{0}\right)$. Let $N\left(t^{0}, x^{0}\right) \subset \Omega$ be an open ball with center $\left(t^{0}, x^{0}\right)$, radius so small and $c=c\left(t^{0}, x^{0}\right)>0$ so small that (i) $L_{(t, x)}^{k}(t, x)$, $k \in I_{0}\left(t^{0}, x^{0}\right)$, are linearly independent on $N\left(t^{0}, x^{0}\right)$, so that (16) has continuous solutions $g_{0}(t, x)=g_{0}\left(t, x ; t^{0}, x^{0}\right), g(t, x)=g\left(t, x ; t^{0}, x^{0}\right)$ on $N\left(t^{0}, x^{0}\right)$ for $\alpha, \beta \in I_{0}\left(t^{0}, x^{0}\right) ; \cdot\left(\right.$ ii) if $\left(t^{0}, x^{0}\right) \notin \Pi^{k}$, then $L^{k}(t, x)<0$ on $N\left(t^{0}, x^{0}\right)$ and if $\left(t^{0}, x^{0}\right) \in \Pi^{k}, k \notin I\left(t^{0}, x^{0}\right)$, then $\left|\dot{L}^{k}\right|>c\left|L_{t}^{k} g_{0}+L_{x}^{k} \cdot g\right|$ on $N\left(t^{0}, x^{0}\right)$; finally (iii) $g_{0}, g$ and $c$ satisfy

$$
c\left|g_{0}\right|, c|g| \leqq \frac{1}{2} \quad \text { on } N\left(t^{0}, x^{0}\right) .
$$

Condition (ii), (16), and the definition of $I_{0}=I_{a 0} \cup I_{b 0}$ imply that $L^{k}(t, x)<0$ on $N\left(t^{0}, x^{0}\right)$ if $\left(t^{0}, x^{0}\right) \notin \Pi^{k}$ and that

$$
\begin{aligned}
\dot{L}^{k}+\varepsilon c\left(L_{t}^{k} g_{0}+L_{x}^{k} \cdot g\right)>0[\text { or }<0] \quad \text { on } \Pi^{k} \cap N\left(t^{0}, x^{0}\right) \\
\text { if } k=\alpha \quad \text { [or } k=\beta]
\end{aligned}
$$

and $\left(t^{0}, x^{0}\right) \in \Pi^{k}, 0<\varepsilon \leqq 1$ (whether or not $k \in I\left(t^{0}, x^{0}\right)$ ).

The set $R=\{(t, x) \in \Omega: I(t, x)$ is not empty $\}$ is closed relative to $\Omega$. Choose a sequence of points $\left(t^{m}, x^{m}\right) \in R, m=1,2, \cdots$, such that $N_{1} \cup$ $N_{2} \cup \cdots$, where $N_{m}=N\left(t^{m}, x^{m}\right)$, is a cover of $R$. Correspondingly, let $c^{m}=c\left(t^{m}, x^{m}\right), g^{m 0}(t, x)=g_{0}\left(t, x ; t^{m}, x^{m}\right)$, and $g^{m}(t, x)=g\left(t, x ; t^{m}, x^{m}\right)$. On the open set $N_{0}=\Omega-R$, let $g^{00}(t, x)=0, g^{0}(t, x)=0, c^{0}=0$; so that

$$
\begin{array}{r}
\dot{L}^{k}+\varepsilon c^{0}\left(L_{t}^{k} \cdot g^{00}+L_{r}^{k} \cdot g^{0}\right)=L^{k}>0[\text { or }<0] \text { on } \Pi^{k} \cap N_{0} \\
\text { if } k=\alpha \quad[\text { or } k=\beta] .
\end{array}
$$

Let $\phi^{0}(t, x), \phi^{1}(t, x), \cdots$, be a continuous partition of unity subordinate to the open cover $N_{0} \cup N_{1} \cup \cdots$, of $\Omega$, and put

$$
h_{0}(t, x)=\sum c^{m} g^{m 0}(t, x) \phi^{m}(t, x), \quad h(t, x)=\sum c^{m} g^{m}(t, x) \phi^{m}(t, x) .
$$

The inequalities (9) are implied by (17) and $g^{00}=0, g^{0}=0$. In order to verify (10), let $\left(t^{0}, x^{0}\right)=\left(t^{m}, x^{m}\right)$ in (18), multiply (18) by $\phi^{m}$, sum with 
respect to $m=1,2, \cdots$, and add the result to (19) multiplied by $\phi^{0}$. In this way, we obtain (10).

Proof of Theorem 3. Consider the system of differential equations

$$
x^{\prime}=[f(t, x)+\varepsilon h(t, x)] /\left[1+\varepsilon h_{0}(t, x)\right],
$$

equivalent to (12). [2, Corollary 3.1, p. 282] implies that there exists a point $\left(t_{\varepsilon}, x_{\varepsilon}\right) \in S$ and a solution $x=x_{\varepsilon}(t)$ of the system (20) such that $x_{\varepsilon}\left(t_{\varepsilon}\right)=x_{\varepsilon}$ and $\left(t, x_{\varepsilon}(t)\right) \in \Omega^{0}$ on its right maximal interval of existence.

From the compactness of $S$, it follows that there exist a $\left(t_{0}, x_{0}\right) \in S$, an $\omega$ on $0<\omega \leqq \infty$, and a sequence $\varepsilon(1)>\varepsilon(t)>\cdots$, such that $\varepsilon(m) \rightarrow 0$ and $x(t)=\lim x_{\varepsilon(m)}(t)$ exists uniformly on compact intervals of $0 \leqq t<\omega$ as $m \rightarrow \infty, x=x(t)$ is a solution of (8), and $0 \leqq t<\omega$ is its right maximal interval of existence; cf. [2, Theorem 3.2, pp. 14-15]. This proves Theorem 3.

Let $F \subset E^{n+1}$ be a convex set with a nonempty interior $F^{0}$. Let $(t, x) \in \partial F$ and $\mathscr{N}(t, x)$ the set of outward normal vectors $\Delta$ to $F$ at $(t, x)$. Then $\mathscr{N}(t, x)$ is a closed, convex cone (i.e., $\Delta_{1}, \Delta_{2} \in \mathscr{N}(t, x)$ implies that $\lambda_{1} \Delta_{1}+$ $\lambda_{2} \Delta_{2} \in \mathscr{N}(t, x)$ for $\left.\lambda_{1}, \lambda_{2} \geqq 0\right)$. A vector $\Delta \in \mathscr{N}(t, x)$ will be called extremal if $\Delta \neq 0$ and the only line segment in $\mathscr{N}(t, x)$ with $\Delta$ as interior point is on the ray $\lambda \Delta, \lambda \geqq 0$. Let $\mathscr{N}_{e}(t, x)$ be the set of unit, extremal, outward normal vectors to $F$ at $(t, x) \in \partial F$.

(A1) $\Omega \subset E^{n+1}$ is open, $f: \Omega \rightarrow E^{n}$ is continuous, and $v=v(t, x)$ is the $(n+1)$-vector

$$
v=v(t, x)=(1, f(t, x)) .
$$

(A2) $F \subset \Omega$ is closed relative to $\Omega$; the interior $F^{0}$ of $F$ is not empty; and, for every $\left(t_{0}, x_{0}\right) \in F$, there is a ball $\Sigma=\Sigma\left(t_{0}, x_{0}\right) \subset \Omega$ with center $\left(t_{0}, x_{0}\right)$ such that $F \cap \Sigma$ is convex.

(A3) Introduce the sets

$$
\left.\mathscr{N}\left[\operatorname{or} \mathscr{N}_{e}\right]=\left\{(t, x, \Delta): \Delta \in \mathscr{N}(t, x) \text { [or } \Delta \in \mathscr{N}_{e}(t, x)\right],(t, x) \in \partial F \cap \Omega\right\},
$$

$\mathscr{N}_{e} \subset \mathscr{N} \subset \Omega \times E^{n+1}$. Define by the projection $P: \mathscr{N} \rightarrow \partial F \cap \Omega$,

$$
P(t, x, \Delta)=(t, x) \text { for }(t, x, \Delta) \in \mathscr{N} \text {. }
$$

Suppose that the set $\mathscr{N}_{e}$, which is closed relative to $\Omega \times E^{n+1}$, can be written as a disjoint union $\mathscr{N}_{e 1} \cup \mathscr{N}_{e 2}$ such that (i) $\mathscr{N}_{e 1}, \mathscr{N}_{e 2}$ are closed relative to $\Omega \times E^{n+1}$; (ii) we have the inequalities

$$
\Delta \cdot v(t, x) \geqq 0 \text { [or } \leqq 0] \text { for }(t, x, \Delta) \in \mathscr{N}_{e 1}\left[\text { or } \mathscr{N}_{e 2}\right. \text { ]; }
$$

(iii) for fixed $(t, x) \in \partial F \cap \Omega$, the closed convex cones $C_{1}(t, x)$ [or $C_{2}(t, x)$ ] generated by $\{\Delta\},(t, x, \Delta) \in \mathscr{N}_{1 e}$ [or $\mathscr{N}_{2 e}$ ] have only $\Delta=0$ in common. (Note that if $\mathscr{N}_{e}(t, x)$ is connected for some $(t, x) \in \partial F \cap \Omega$, then $\mathscr{N}_{e}(t, x) \subset$ $\mathscr{N}_{j e}$ for $j=1$ or $j=2$.) 
(A4) Let $Q \subset \partial F \cap \Omega$ be the set-theoretic difference

$$
Q=P \mathscr{N}_{1 e}-P \mathscr{N}_{2 e}
$$

$S$ a nonempty compact subset of $F^{0} \cup Q$ such that $S \cap Q$ is a retract of $Q$ but not of $S$.

TheOREM 4. Assume (A1)-(A4). Then there is a point $\left(t_{0}, x_{0}\right) \in S$ and $a$ solution of the initial value problem

$$
x^{\prime}=f(t, x), \quad x\left(t_{0}\right)=x_{0},
$$

such that $(t, x(t)) \in F$ on its right maximal interval of existence.

The proof parallels that of Theorem 3 . We first obtain

LemMa 2. Assume (A1)-(A4). Then there exists a continuous $u: \Omega \rightarrow E^{n+1}$ with the properties that $|u(t, x)| \leqq \frac{1}{2}$ and

$$
\Delta \cdot u(t, x)>0[\text { or }<0] \text { for }(t, x, \Delta) \in \mathscr{N}_{e 1}\left[\text { or } \mathscr{N}_{e 2}\right]
$$

Proof of Lemma 2. Let $\left(t^{0}, x^{0}\right) \in \partial F \cap \Omega$. Since the closed convex cones $C_{1}\left(t^{0}, x^{0}\right), C_{2}\left(t^{0}, x^{0}\right)$ in (A3) have only the point $\Delta=0$ in common, there exists an $(n+1)$-vector $u\left(t^{0}, x^{0}\right)$ such that $\left|u\left(t^{0}, x^{0}\right)\right| \leqq \frac{1}{2}$ and $\Delta \cdot u\left(t^{0}, x^{0}\right)>0$ [or $<0$ ] for $0 \neq \Delta \in C_{1}\left(t^{0}, x^{0}\right)$ [or $C_{2}\left(t^{0}, x^{0}\right)$ ]. Also, since the sets $\mathscr{N}_{e}, \mathscr{N}_{e 1}, \mathscr{N}_{e 2}$ are closed relative to $\Omega \times E^{n+1}$, it follows that, for a sufficiently small open neighborhood $N=N\left(t^{0}, x^{0}\right) \subset \Omega$ of $\left(t^{0}, x^{0}\right)$, $\Delta \cdot u\left(t^{0}, x^{0}\right)>0$ [or $<0$ ] for $0 \neq \Delta \in C_{1}(t, x)$ [or $C_{2}(t, x)$ ], $(t, x) \in \partial F \cap N$. The proof can now be completed by using a partition of unity relative to an open cover $\left\{N_{m}=N\left(t^{m}, x^{m}\right)\right\}, m=1,2, \cdots$, of $\partial F \cap \Omega$ and $N_{0}=\Omega-\partial F$, as in the proof of Lemma 1 .

Proof of Theorem 4. Let $u=u(t, x)$ be given in Lemma 2 and write $u=\left(h_{0}, h\right)$, where $h_{0}: \Omega \rightarrow E^{1}, h: \Omega \rightarrow E^{n}$. For $0<\varepsilon \leqq 1$, consider the differential equation

$$
x^{\prime}=f_{\varepsilon}(t, x) \equiv[f(t, x)+\varepsilon h(t, x)] /\left[1+\varepsilon h_{0}(t, x)\right] .
$$

If $v_{\varepsilon}=v_{\varepsilon}(t, x)=\left(1, f_{\varepsilon}(t, x)\right)$, then, by (22) and (25),

$$
\Delta \cdot v_{\varepsilon}(t, x)>0[\text { or }<0] \text { for }(t, x, \Delta) \in \mathscr{N}_{1 e}\left[\text { or } \mathscr{N}_{2 e}\right] \text {, }
$$

since $\Delta \cdot v_{\varepsilon}=(\Delta \cdot v+\varepsilon \Delta \cdot u) /\left(1+\varepsilon h_{0}\right)$.

If $\Delta \in \mathscr{N}_{e}\left(t^{0}, x^{0}\right),\left(t^{0}, x^{0}\right) \in \partial F \cap \Omega$, and $V \cdot \Delta<0$, then the point $\left(t^{0}, x^{0}\right)+$ $h V \notin F$ for small $-h>0$. In particular, $\Delta \cdot v_{\varepsilon}\left(t^{0}, x^{0}\right)<0$ when $\left(t^{0}, x^{0}, \Delta\right) \in$ $\mathscr{N}_{2 e}$ implies that $\left(t^{0}, x^{0}\right) \in P \mathscr{N}_{2 e}$ is not an egress point of $F^{0}$ relative to the differential equation (26). In fact, the set of egress points of $F^{0}$ relative to (26) is $Q$ in (23), and every egress point is a strict egress point; cf. e.g., the proof of [2, Lemma 3.1, p. 281]. 
We claim that there exists a $\left(t_{\varepsilon}, x_{\varepsilon}\right) \in S$ such that (26) has a solution $x=x_{\varepsilon}(t)$ satisfying $x_{\varepsilon}\left(t_{\varepsilon}\right)=x_{\varepsilon}$ and $\left(t, x_{\varepsilon}(t)\right) \in F^{0}$ on its right maximal interval of existence. This follows from a theorem of Ważewski (cf. [2, Theorem 2.1, p. 279]) if the solutions of initial value problems associated with (26) are unique. If this uniqueness property does not hold, we can obtain the same conclusion by approximating $f_{\varepsilon}$, for fixed $\varepsilon>0$, by smooth functions; cf. the proof of [2, Corollary 3.1, p. 282]. Finally, we obtain Theorem 4 by using a suitable sequence $\varepsilon=\varepsilon(1)>\varepsilon(2)>\cdots, \varepsilon(m) \rightarrow \infty$, as in the proof of Theorem 3 .

\section{REFERENCES}

1. H. Brezis, On a characterization of flow-invariant sets, Comm. Pure Appl. Math. 23 (1970), 261-263. MR 41 \#2161.

2. P. Hartman, Ordinary differential equations, Wiley, New York, 1964. MR 30 \#1270.

Department of Mathematics, The Johns Hopkins University, Baltimore, MaryLAND 21218 